medRxiv preprint doi: https://doi.org/10.1101/2021.12.05.21267310; this version posted December 11,2021 . The copyright holder for this preprint (which was not certified by peer review) is the author/funder, who has granted medRxiv a license to display the preprint in perpetuity.

It is made available under a CC-BY-NC-ND 4.0 International license .

\section{Detection of acne by deep learning object detection}

\author{
Amandip Sangha ${ }^{1}$ \\ Mohammad Rizvi ${ }^{1,2}$ \\ ${ }^{1}$ Askin \\ ${ }^{2}$ Oslo University Hospital Rikshospitalet \\ E-mail corresponding author: \\ amandip.sangha@askin.no
}

\section{Key Points}

Question Can deep learning-based acne detection models trained on a small data set of publicly available photos of patients with acne achieve high prediction accuracy?

Findings We find that it is possible to train a reasonably good object detection model on a small, annotated data set of acne photos using standard deep learning architectures.

Meaning Deep learning-based object detection models for acne detection can be a useful decision support tools for dermatologists treating acne patients in a digital clinical practice. It can prove a particularly useful tool for monitoring the time evolution of the acne disease state over prolonged time during follow-ups, as the model predictions give a quantifiable and comparable output for photographs over time. This is particularly helpful in teledermatological consultations, as a prediction model can be integrated in the patient-doctor remote communication.

\section{Abstract}

\section{Importance}

State-of-the art performance is achieved with a deep learning object detection model for acne detection. There is little current research on object detection in dermatology and acne in particular. As swoht: this work is early in this field and achichieves state of the art performance.

\title{
Objective
}

Train an object detection model on a publicly available data set of acne photos.

\section{Design, Setting, and Participants}

A deep learning model is trained with cross validation on a data set of facial acne photos.

\section{Main Outcomes and Measures}

Object detection models for detecting acne for single-class (acne) and multi-class (four severity levels). We train and evaluate the models using standard metrics such as mean average precision (mAP). Then we manually evaluate the model predictions on the test set, and calculate accuracy in terms of precision, recall, $F 1$, true and false positive and negative detections.

\section{Results}

We achieve state-of-the art mean average precision mAP@0.5 value of 37.97 for the single class acne detection task, and 26.50 for the 4 -class acne detection task. Moreover, our manual evaluation shows that the single class detection model performs well on the validation set, achieving true positive $93.59 \%$, precision 96.45 $\%$ and recall $94.73 \%$.

\section{Conclusions and Relevance}

We are able to train a high-accuracy acne detection model using only a small publicly available data set of facial acne. Transfer learning on the pre-trained deep learning model yields good accuracy and high degree of transferability to patient submitted photographs. We also note that the training of standard architecture object detection models has given significantly better accuracy than more intricate and bespoke neural network architectures in the existing research literature.

\section{Introduction}

Acne is a chronic inflammatory disease of the skin and is among the most common skin conditions ${ }^{5}$ in the world. The Global Burden of Disease study (2010) found that acne was the eight most prevalent disease globally, estimated to affect $9.4 \%$ of the global population'. More than $95 \%$ of teenage boys and more than $85 \%$ of teenage girls are affected, and more than $20 \%$ of those affected peer review and should not be used to guide clinical practice. 
medRxiv preprint doi: https://doi.org/10.1101/2021.12.05.21267310; this version posted December 11, 2021. The copyright holder for this preprint (which was not certified by peer review) is the author/funder, who has granted medRxiv a license to display the preprint in

It is made available under a CC-BY-NC-ND 4.0 International license .

have moderate to severe acne, and $50 \%$ continue to suffer from acne in their adulthood.

The pathogenesis of acne is multifaceted. Hyperkeratinization is the excessive accumulation of dead skin cells causing a blockage of hair follicles. Abnormal keratinization of the infundibular epitheleum causes an obstruction of sebaceous follicles. Androgenic stimulation of sebaceous glands increase secretion. Microbial colonization of pilosebaceous units by the bacteria cutibacterium acnes contributes to inflammation. The clinical and thus visual presentation of acne results in

- comedones - whiteheads and blackheads (mild)

- pustules

- papules

- nodules and cysts (severe)

We train a deep learning object detection model on a publicly available image set to predict acne in photos. Our model achieves state of the art accuracy with an existing deep neural network architecture and no special preprocessing or hyperparameter evolution. We train on both single class (acne) detection and multi class (severity levels 1-4).

\section{Methods}

\section{Data}

We train the model on publicly available data set ACNEO $4^{3}$ by Wu et al (ref). This data set consists of 1457 photographs of patients. The photos are taken of the patients' faces at an approximate angle of 70 degrees from the front. The photos have been manually annotated by experts, so that the acne lesions in the photographs have been enclosed in bounding boxes, a total number of 18983 bounding boxes.

We split the data set randomly into a training-test split of $80 \%$ for training (1165 photos, 14879 lesion bounding boxes) and the remaining $20 \%$ for testing (292 photos, 4104 lesion bounding boxes).

The ACNEO4 dataset is used for training and validating the model, i.e. building the deep learning model and deriving model validation metrics such as precision, recall and mAP. Secondarily, we employ a small data set consisting of photographs submitted by patients in our clinical practice. We use only completely anonymous photographs where the person is unidentifiable.

\section{Deep learning object detection model}

Object detection is the branch of computer vision that deals with the identification of specific objects within digital photos. Deep learning-based models have achieved great success in the field of object detection recently and is now the dominant technique. These models are deep convolutional neural networks ${ }^{17}$ which are trained on large, annotated image datasets to predict rectangular bounding boxes around objects of interest in the images.

We use the model YOLOv5 which is a convolutional neural network consisting of 773 layers and 141.8 million parameters. Recall that the YOLO-family of object detection models is an example of a single-stage detector where a single neural network is responsible for predicting bounding boxes and class probabilities from full images directly in one evaluation. Object detection is framed as a regression problem of spatially separated bounding rectangles. This is in contrast to other prominent object detection models that are two-stage detectors, for example the Fast RCNN family of models which employ one neural network for proposing regions of interest and another neural network for predicting bounding boxes within the proposed regions.

The YOLOv5 $\mathrm{P} 6^{15}$ model for object detection is a single-stage detector that has been pre-trained on the $\mathrm{COCO}^{9}$ data set.

\begin{tabular}{|l|l|}
\hline Model & YOLOv5x6 \\
\hline Image size (px) & 1280 \\
\hline $\begin{array}{l}\text { mAPval } \\
\text { 0.5:0.95 }\end{array}$ & 54.4 \\
\hline $\begin{array}{l}\text { mAPtest } \\
\text { 0.5:0.95 }\end{array}$ & 54.4 \\
\hline $\begin{array}{l}\text { mAPval } \\
\text { 0.5 }\end{array}$ & 72.0 \\
\hline $\begin{array}{l}\text { Speed } \\
\text { v100(m) }\end{array}$ & 22.4 \\
\hline $\begin{array}{l}\text { params } \\
\text { (M) }\end{array}$ & 141.8 \\
\hline $\begin{array}{l}\text { FLOPS } \\
\text { (B) }\end{array}$ & 222.9 \\
\hline
\end{tabular}


medRxiv preprint doi: https://doi.org/10.1101/2021.12.05.21267310; this version posted December 11, 2021. The copyright holder for this preprint (which was not certified by peer review) is the author/funder, who has granted medRxiv a license to display the preprint in

It is made available under a CC-BY-NC-ND 4.0 International license .

We use default hyperparameter values: learning rate 0.01 , SGD momentum 0.937 and weight decay 0.0005 , and conduct no hyperparameter evolution.

Recall the standard evaluation metrics of the object detection task. Intersection over Union, $\mathrm{IOU}$, is the degree of overlap of two enclosed pixel regions. This clearly measures the accuracy of a predicted rectangle.

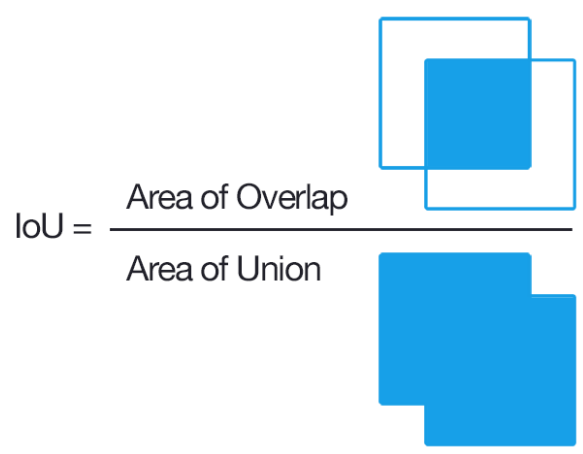

Precisionand recall should be familiar notions from binary classification. Precision is the fraction of correct detections among all detections

$$
=\frac{\text { precision }}{\text { true positive }+ \text { false positive }}
$$

Recall is the fraction of correct detections retrieved

$$
\text { recall }=\frac{\text { true positive }}{\text { true positive }+ \text { false negative }}
$$

By plotting the precision and recall as a curve in the plane, one gets the Precision-Recall-curve. The area under this curve is referred to as average precision(AP). The mean average precision (mAP) refers to averaging the AP over all classes. When one refers to MAP@0.5, one has fixed an loUthreshold of 0.5 , meaning that one only considers predictions where the loU metric has value greater than 0.5. The mAP value is the main performance metric used in training object detection models.

The process of training the model is the standard machine learning flow. A large portion of the data set, e.g. $80 \%$ is taken as training samples, while the remaining $20 \%$ is taken as test samples. We replicate this process in five separate iterations, meaning that we do random sampling into 80-20 split 5 times. Thus we conduct training and testing in 5 different iterations. This is to minimize any bias which would result from having an uneven data split by chance.

\section{Evaluation of model performance}

Our main objective in this work is to train an object detection model. In addition to the standard evaluation metrics such as loU and mAP, we do the following manual assessment of the predictions on the test set:

- Number of detections by human (this is considered ground truth)

- Number of correct detections by model (true positive)

- Number of incorrect detections by model (false positive)

- Number of missing detections by model (false negative)

We also remark that for a dermatologist, the value of the object detection model as a decision support tool does not depend strictly on the model's ability to give optimal bounding boxes with minimal pixel areas around each acne lesion. For the dermatologist, a fairly accurate bounding box around the acne lesions will suffice. This practical aspect is important to have in mind when evaluating such models. Of course, the loU and $\mathrm{mAP}$ metrics are important in model training and validation, but in practical visual validation the minimization of the area of the bounding box is not essential to model performance and quality.

Hence we work with the following manual evaluation criteria:

\begin{tabular}{|l|l|}
\hline Metric & Description \\
\hline $\begin{array}{l}\text { Number of } \\
\text { detections by } \\
\text { model }\end{array}$ & $\begin{array}{l}\text { Number of acne lesions } \\
\text { detected by the trained } \\
\text { model }\end{array}$ \\
\hline $\begin{array}{l}\text { Number of } \\
\text { detections by } \\
\text { human }\end{array}$ & $\begin{array}{l}\text { Number of acne lesions } \\
\text { detected by the human } \\
\text { dermatologist }\end{array}$ \\
\hline $\begin{array}{l}\text { Correct detections } \\
\text { (true positive) by } \\
\text { model }\end{array}$ & $\begin{array}{l}\text { Number of acne lesions } \\
\text { correctly detected by } \\
\text { model, as judged by } \\
\text { dermatologist }\end{array}$ \\
\hline $\begin{array}{l}\text { Wrong detections } \\
\text { (false positive) by } \\
\text { model }\end{array}$ & $\begin{array}{l}\text { Number of acne lesions } \\
\text { incorrectly detected by } \\
\text { model, as judged by } \\
\text { dermatologist }\end{array}$ \\
\hline
\end{tabular}


medRxiv preprint doi: https://doi.org/10.1101/2021.12.05.21267310; this version posted December 11, 2021. The copyright holder for this preprint (which was not certified by peer review) is the author/funder, who has granted medRxiv a license to display the preprint in

It is made available under a CC-BY-NC-ND 4.0 International license .

\begin{tabular}{|l|l|}
\hline $\begin{array}{l}\text { Missed detections } \\
\text { (false negative) by } \\
\text { model }\end{array}$ & $\begin{array}{l}\text { Number of acne lesions } \\
\text { not detected by model, } \\
\text { as judged by } \\
\text { dermatologist }\end{array}$ \\
\hline
\end{tabular}

\section{Results}

\section{Single class detection}

The single class ("acne") detection model achieves a maximum mAP@0.5 value 37.97 after only 5 epochs. We conduct a 5 -fold cross validation training with an image size of $640 \times 640$ pixels, and a training-test split of 80-20. The training is run for 100 epochs with a batch size of 16 and the SGD optimizer. Each epoch took approximately 2 minutes on an Nvidia Tesla V100 GPU with 16GB VRAM.

\begin{tabular}{|l|l|}
\hline Best mAP@0.5 & Best epoch \\
\hline 37.97 & 5 \\
\hline
\end{tabular}

\begin{tabular}{|l|l|l|}
\hline $\begin{array}{l}\text { Cross } \\
\text { validation fold }\end{array}$ & $\begin{array}{l}\text { Best } \\
\text { mAP@0.5 }\end{array}$ & Best epoch \\
\hline 1 & 36.81 & 8 \\
\hline 2 & 37.97 & 5 \\
\hline 3 & 35.22 & 4 \\
\hline 4 & 35.41 & 22 \\
\hline 5 & 36.53 & 20 \\
\hline
\end{tabular}

The following evaluation metrics on our clinical data set are achieved using the YOLOv5 model combined with visual inspection of the dermatologist as described above.

\begin{tabular}{|l|l|l|}
\hline Measure & Value & Explanation \\
\hline $\begin{array}{l}\text { True } \\
\text { positive }\end{array}$ & $\begin{array}{l}93.59 \\
\%\end{array}$ & Correct detections \\
\hline $\begin{array}{l}\text { False } \\
\text { positive }\end{array}$ & $\begin{array}{l}3.44 \\
\%\end{array}$ & False detections \\
\hline $\begin{array}{l}\text { False } \\
\text { negative }\end{array}$ & $\begin{array}{l}5.20 \\
\%\end{array}$ & Missed detections \\
\hline Precision & $\begin{array}{l}96.45 \\
\%\end{array}$ & $\begin{array}{l}\text { Fraction of correct } \\
\text { detections among all } \\
\text { detections: }\end{array}$ \\
\hline
\end{tabular}

\begin{tabular}{|l|l|l|}
\hline & & $\frac{1}{\text { true positive }}$ \\
\hline Recall & $\begin{array}{l}94.73 \\
\%\end{array}$ & $\begin{array}{l}\text { Fraction of correct } \\
\text { detections retrieved: } \\
\frac{\text { true positive }}{\text { true positive }+ \text { false negative }}\end{array}$ \\
\hline F1 & $\begin{array}{l}95.58 \\
\%\end{array}$ & $\begin{array}{l}\text { Harmonic mean of precision } \\
\text { and recall: }\end{array}$ \\
& $\frac{2 \cdot \text { precision } \cdot \text { recall }}{\text { precision }+ \text { recall }}$ \\
\hline
\end{tabular}

Note that we do not measure true negative (correctly detect non-acne) because healthy skin is not explicitly detected by the model and hence is not relevant.

\section{Multi class detection}

The multi class detection model, where the classes are severity levels $1-4$, achieves a maximum mAP@0.5 value 26.50 after 20 epochs. Also, here we use an image size of $640 \times 640$ pixels, and a training-test split of 80-20. The training is again run for 100 epochs total with a batch size of 16 and the SGD optimizer. Each epoch took approximately 2 minutes on an Nvidia Tesla V100 GPU with 16GB VRAM.

\begin{tabular}{|l|l|}
\hline Best mAP@0.5 & Best epoch \\
\hline 26.50 & 20 \\
\hline
\end{tabular}

\section{Discussion}

\section{Model strengths}

The YOLOv 5 object detection model trained on the ACNEO4 has a number of strengths. It is able to be well trained on both the single class task (acne) and the multi class task $(0,1,2,3)$ where the classes are the varying severity levels. As such, the model is able to learn the main different types of acne: papules, pustules, nodules and cysts. Moreover, the model also seems to correctly learn excoriations and post inflammatory hyperpigmentation and scarring. The model detects acne on all body parts, not only the face. The model is also robust for different skin tones. Blurry images do not disturb the model, and the 
medRxiv preprint doi: https://doi.org/10.1101/2021.12.05.21267310; this version posted December 11, 2021. The copyright holder for this preprint (which was not certified by peer review) is the author/funder, who has granted medRxiv a license to display the preprint in

It is made available under a CC-BY-NC-ND 4.0 International license .

model accepts varying zoom levels and lighting conditions also.

\section{Model weaknesses}

The trained model does sometimes tend to confuse certain body regions for acne lesions. These body regions are typically the creese of the lips, nostrils, nipples and sometimes hair follicles. The reason is evidently that these body parts do have similar shape and color to inflammatory acne lesions, i.e. having a red or pink color. Another source of confusion are hyperpigmented lesions, as acne too is often accompanied with postinflammatory hyperpigmentation. We hypothesize that these confusions can be alleviated by introducing more photos of such artefacts without acne, so that the model can learn by seeing more complicated true negative samples.

\section{Conclusions}

Our object detection model is trained on a small scale publicly available data set and is proving to be useful in our clinical practice. We have shown that one can achieve fairly accurate models by simple means.

The mAP metrics have much room for improvement, but the model is still useful at this performance level. We also note that the training of standard architecture object detection models has given significantly better accuracy than the more intricate custom approaches in the existing research literature concerning acne detection in photographs

\section{References}

1. Tan JK, Bhate K. A global perspective on the epidemiology of acne. Br J Dermatol. 2015 Jul; 172 Suppl 1:3-12. doi: 10.1111/bjd.13462. PMID: 25597339.

2. Pagliarello C, Di Pietro C, Tabolli S. A comprehensive health impact assessment and determinants of quality of life, health and psychological status in acne patients. G Ital Dermatol Venereol. 2015 Jun;150(3):303-8. PMID: 25946674.

3. X. Wu et al., Joint Acne Image Grading and Counting via Label Distribution Learning, 2019 IEEE/CVF International Conference on Computer Vision (ICCV), Seoul, Korea (South), 2019, pp. 10641-10650, doi: 10.1109/ICCV.2019.01074.

4. J. Deng, W. Dong, R. Socher, L. Li, Kai Li and Li Fei-Fei, ImageNet: A large-scale hierarchical image database, 2009 IEEE Conference on Computer Vision and Pattern Recognition, Miami, FL, 2009, pp. 248-255, doi: 10.1109/CVPR.2009.5206848

5. Tuchayi, S., Makrantonaki, E., Ganceviciene, R. et al. Acne vulgaris. Nat Rev Dis Primers 1, 15029 (2015). https://doi.org/10.1038/nrdp.2015.29

6. Russakovsky, O., Deng, J., Su, H. et al. ImageNet Large Scale Visual Recognition Challenge. Int J Comput Vis 115, 211-252 (2015).

7. S. Ren, K. He, R. Girshick and J. Sun, "Faster R-CNN: Towards Real-Time Object Detection with Region Proposal Networks," in IEEE Transactions on Pattern Analysis and Machine Intelligence, vol. 39, no. 6, pp. 1137-1149, 1 June 2017 , doi: 10.1109/TPAMI.2016.2577031.

8. K. He, X. Zhang, S. Ren and J. Sun, "Deep Residual Learning for Image Recognition," 2016 IEEE Conference on Computer Vision and Pattern Recognition (CVPR), Las Vegas, NV, 2016, pp. 770-778, doi: 10.1109/CVPR.2016.90.

9. Lin TY, Maire M, Belongie S, Hays J, Perona P, Ramanan D, Dollár P, Zitnick L. Microsoft COCO: Common Objects in Context. Computer Vision -- ECCV 2014, Springer International Publishing, pp.740755

10. R. Padilla, S. L. Netto and E. A. B. da Silva, "A Survey on Performance Metrics for Object-Detection Algorithms» 2020 International Conference on Systems, Signals and Image Processing (IWSSIP), 
medRxiv preprint doi: https://doi.org/10.1101/2021.12.05.21267310; this version posted December 11, 2021. The copyright holder for this preprint (which was not certified by peer review) is the author/funder, who has granted medRxiv a license to display the preprint in It is made available under a CC-BY-NC-ND 4.0 International license.

Niteroi, Brazil, 2020, pp. 237-242, doi: 10.1109/IWSSIP48289.2020.9145130.

11. J. Redmon, S. Divvala, R. Girshick, A. Farhadi. «You Only Look Once: Unified, Real-Time Object Detection». 2016, arXiv:1506.02640 [cs.CV]

12. J. Redmon, A. Farhadi. «YOLO9000: Better, Faster, Stronger». 2016, arXiv: 1612.08242 [cs.CV]

13. J. Redmon, A. Farhadi. «YOLOv3: An Incremental Improvement». 2018, arXiv:1804.02767 [cs.CV]

14. A. Bochkovskiy, C-Y. Wang, H-Y. M. Liao. "YOLOv4: Optimal Speed and Accuracy of Object Detection». 2020, arXiv:2004.10934 [cs.CV]

15. G. Jocher et al., ultralytics/yolov5: v4.0 nn.SiLU() activations, Weights \& Biases logging, PyTorch Hub integration. 2021, doi:10.5281/zenodo.4418161

16. Min, Kyungseo \& Lee, Gun-Hee \& Lee, Seong-Whan. (2021). ACNet: Mask-Aware Attention with Dynamic Context Enhancement for Robust Acne Detection.

17. LeCun, Y., Bengio, Y. \& Hinton, G. Deep learning. Nature521, 436-444 (2015). 